\title{
Almansi Theorem and Mean Value Formula for Quaternionic Slice-regular Functions
}

\author{
Alessandro Perotti*[0
}

\begin{abstract}
We prove an Almansi Theorem for quaternionic polynomials and extend it to quaternionic slice-regular functions. We associate to every such function $f$, a pair $h_{1}, h_{2}$ of zonal harmonic functions such that $f=h_{1}-\bar{x} h_{2}$. We apply this result to get mean value formulas and Poisson formulas for slice-regular quaternionic functions.
\end{abstract}

Mathematics Subject Classification. Primary 30G35, Secondary 31B30, 33C50, 33C55.

Keywords. Quaternionic polynomials, Slice-regular functions, Zonal harmonics, Almansi theorem, Mean value property, Poisson formula.

\section{Introduction}

In this note we prove a quaternionic Almansi-type Theorem for polynomials with quaternionic coefficients and, more generally, for slice-regular functions of a quaternionic variable (see Sect. 3 for definitions and references about this function theory).

We show that to every slice-regular function $f$ defined on a domain $\Omega$ of the skew field $\mathbb{H}$ of quaternions, it is possible to associate two uniquely defined harmonic functions $h_{1}, h_{2}$ such that $f(x)=h_{1}(x)-\bar{x} h_{2}(x)$ on $\Omega$. This result allows to apply well-known results of harmonic function theory on $\mathbb{R}^{4}$ to obtain a mean value property for slice-regular functions and a Poisson-type formula over three-dimensional spheres. An interesting aspect of these last results is their validity over any sphere in $\Omega$, without requiring any symmetry with respect to the real axis.

This work was supported by GNSAGA of INdAM, and by the grants "Progetto di Ricerca INdAM, Teoria delle funzioni ipercomplesse e applicazioni", and PRIN "Real and Complex Manifolds: Topology, Geometry and holomorphic dynamics" of Ministero dell'università e della ricerca.

This article is part of the Topical Collection on ISAAC 12 at Aveiro, July 29-August 2, 2019, edited by Swanhild Bernstein, Uwe Kaehler, Irene Sabadini, and Franciscus Sommen.

${ }^{*}$ Corresponding author. 
An Almansi-type Theorem for slice-regular functions on real Clifford algebras $\mathbb{R}_{n}$ (also called slice-monogenic functions [3]) has been proved in [14]. The quaternionic algebra can be identified with the Clifford algebra $\mathbb{R}_{2}$. However, slice-monogenic functions are naturally defined on the paravector space $\mathbb{R}^{n+1}$. Therefore the results of [14] for $n=2$ are related to harmonic function theory in $\mathbb{R}^{3}$ instead of the one in $\mathbb{R}^{4} \simeq \mathbb{H}$. The quaternionic results obtained in the present paper present some similarities with those obtained in [14] for $n=4$, since slice-regular functions on $\mathbb{R}_{3}$, as those on $\mathbb{H}$, are linked to harmonic functions of four real variables (see [13]).

We recall that similar Almansi-type decompositions have been established in other settings, for example in Clifford analysis [10], for Dunkl operators [15] and in the umbral calculus setting [4].

The paper is structured in the following way. In Sect. 2 we prove the Almansi decomposition for quaternionic polynomials, then in Sect. 3 we recall the basic notions of slice function theory on $\mathbb{H}$ and we prove the quaternionic Almansi Theorem for slice-regular functions. Finally, in Sect. 4 we deduce the mean value formulas and the Poisson formula for slice-regular functions.

\section{An Almansi Decomposition for Quaternionic Polynomials}

We consider quaternionic polynomials of the form

$$
P(x)=\sum_{k=0}^{d} x^{k} a_{k} \quad\left(a_{k} \in \mathbb{H}\right),
$$

with quaternionic coefficients on the right and quaternionic variable $x=$ $x_{0}+i x_{1}+j x_{2}+k x_{3}$. It is well-known (see, e.g., [13, Theorem 3.6.3]) that the four real components of these polynomials, considered as functions of the four real variables $x_{0}, x_{1}, x_{2}, x_{3}$, are biharmonic with respect to the standard Laplacian $\Delta$ of $\mathbb{R}^{4}$, i.e., $\Delta^{2} P=0$ on $\mathbb{R}^{4}$.

We recall the classical result of Emilio Almansi, published in 1899 [1], which dealt more generally with polyharmonic functions on $\mathbb{R}^{n}$.

Theorem 1. (Almansi [1]). If $\Delta^{p} u=0$ on a starlike domain $D \subseteq \mathbb{R}^{n}$ with centre 0 , then there exist unique harmonic functions $u_{0}, \ldots, u_{p-1}$ in $D$ such that

$$
u(x)=u_{0}(x)+|x|^{2} u_{1}(x)+\cdots+|x|^{2 p-2} u_{p-1}(x) \quad \text { for } x \in D .
$$

Almansi's Theorem implies that the four real components of a quaternionic polynomial can be expressed in terms of pairs of harmonic functions. Observe that every polynomial $P$ is more than biharmonic, since it belongs to the kernel of a third order operator. If $\bar{\partial}_{C R F}$ denotes the Cauchy-RiemannFueter operator

$$
\bar{\partial}_{C R F}=\frac{1}{2}\left(\frac{\partial}{\partial x_{0}}+i \frac{\partial}{\partial x_{1}}+j \frac{\partial}{\partial x_{2}}+k \frac{\partial}{\partial x_{3}}\right),
$$

then $\bar{\partial}_{C R F} \Delta P=0$ (this is an expression of the generalized Fueter's Theorem). Using the symmetry properties of quaternionic powers $x^{k}$, we can refine the decomposition given by Almansi's Theorem. 
Let $\mathbb{B}$ be the open unit ball in $\mathbb{R}^{4}$ and let $\mathbb{S}^{3}=\partial \mathbb{B}$ the three-dimensional unit sphere. Let $\mathcal{Z}_{k}(x, a)$ denote the real four-dimensional (solid) zonal harmonic of degree $k$ and pole $a \in \mathbb{S}^{3}$ (see, e.g., [2, Ch.5]). From the uniqueness properties of zonal harmonics and their invariance with respect to fourdimensional rotations, it holds $\mathcal{Z}_{k}(x, a)=\mathcal{Z}_{k}(x \bar{a}, 1)$ for every $x \in \mathbb{H}$ and $a \in \mathbb{S}^{3}$. Let $\widetilde{\mathcal{Z}}_{k}(x)$ be the real-valued zonal harmonic defined on $\mathbb{H} \simeq \mathbb{R}^{4}$ by

$$
\left\{\begin{array}{l}
\widetilde{\mathcal{Z}}_{k}(x):=\frac{1}{k+1} \mathcal{Z}_{k}(x, 1) \quad \text { for any } k \geq 0, \\
\widetilde{\mathcal{Z}}_{-1}:=0
\end{array}\right.
$$

Remark 1. The restriction of $\widetilde{\mathcal{Z}}_{k}(x)$ to the unit sphere $\mathbb{S}^{3}$ is equal to the Gegenbauer (or Chebyshev of the second kind) polynomial $C_{k}^{(1)}\left(x_{0}\right)$, where $x_{0}=\operatorname{Re}(x)$.

We recall the following result from [13, Corollary 3.6.7]. It gives the zonal harmonics decomposition of quaternionic powers.

Proposition 1. ([13]). For every $k \in \mathbb{N}$, it holds

$$
x^{k}=\widetilde{\mathcal{Z}}_{k}(x)-\bar{x} \widetilde{\mathcal{Z}}_{k-1}(x) \quad \forall x \in \mathbb{H} .
$$

Example 1. The first four zonal harmonics $\widetilde{\mathcal{Z}}_{k}$ are

$$
\left\{\begin{array}{l}
\widetilde{\mathcal{Z}}_{0}(x)=1, \\
\widetilde{\mathcal{Z}}_{1}(x)=2 x_{0}, \\
\widetilde{\mathcal{Z}}_{2}(x)=3 x_{0}^{2}-x_{1}^{2}-x_{2}^{2}-x_{3}^{2}, \\
\widetilde{\mathcal{Z}}_{3}(x)=4 x_{0}\left(x_{0}^{2}-x_{1}^{2}-x_{2}^{2}-x_{3}^{2}\right) .
\end{array}\right.
$$

In the next statement we will consider polynomials in the four real variables $x_{0}, x_{1}, x_{2}, x_{3}$ of the form

$$
H(x)=\sum_{k=0}^{d} \widetilde{\mathcal{Z}}_{k}(x) a_{k}
$$

with quaternionic coefficients $a_{k}$. All these harmonic polynomials have an axial symmetry with respect to the real axis. For every orthogonal transformation $T$ of $\mathbb{H} \simeq \mathbb{R}^{4}$ that fixes 1 , it holds $H \circ T=H$. These polynomials will be called zonal harmonic polynomials with pole 1 .

From formula (1) we easily deduce the Almansi Theorem for quaternionic polynomials, which was already stated in [12].

Theorem 2. Let $P \in \mathbb{H}[X]$ have degree $d \geq 1$. There exist two zonal harmonic polynomials $h_{1}, h_{2}$ with pole 1 , of degrees $d$ and $d-1$ respectively, such that

$$
P(x)=h_{1}(x)-\bar{x} h_{2}(x) \quad \forall x \in \mathbb{H} .
$$

The restrictions of $h_{1}$ and $h_{2}$ to the unit sphere $\mathbb{S}^{3}$ are $\mathbb{H}$-valued spherical harmonics depending only on $x_{0}=\operatorname{Re}(x)$ 
Proof. Let $P(x)=\sum_{k=0}^{d} x^{k} a_{k}$. Formula (2) follows immediately from (1) by setting

$$
h_{1}(x)=\sum_{k=0}^{d} \widetilde{\mathcal{Z}}_{k}(x) a_{k} \quad \text { and } \quad h_{2}(x)=\sum_{k=0}^{d-1} \widetilde{\mathcal{Z}}_{k}(x) a_{k+1} .
$$

These formulas imply immediately the last statement.

Example 2. Let $P(x)=x^{2}=\left(x_{0}^{2}-x_{1}^{2}-x_{2}^{2}-x_{3}^{2}\right)+2 x_{0} x_{1} i+2 x_{0} x_{2} j+2 x_{0} x_{3} k$. Then $h_{1}(x)=\widetilde{\mathcal{Z}}_{2}=3 x_{0}^{2}-x_{1}^{2}-x_{2}^{2}-x_{3}^{2}$ and $h_{2}(x)=\widetilde{\mathcal{Z}}_{1}=2 x_{0}$.

Example 3. Consider the polynomial

$$
P(x)=x^{3}-x^{2}(i+j+1)+x(i+j+k)-k .
$$

The Almansi decomposition of $P$ is $P(x)=h_{1}(x)-\bar{x} h_{2}(x)$, with quaternionicvalued harmonic polynomials

$$
\begin{aligned}
h_{1}(x)= & \widetilde{\mathcal{Z}}_{3}(x)-\widetilde{\mathcal{Z}}_{2}(x)(i+j+1)+\widetilde{\mathcal{Z}}_{1}(x)(i+j+k)-\widetilde{\mathcal{Z}}_{0}(x) k \\
= & \left(4 x_{0}^{3}-4 x_{0} x_{1}^{2}-4 x_{0} x_{2}^{2}-4 x_{0} x_{3}^{2}-3 x_{0}^{2}+x_{1}^{2}+x_{2}^{2}+x_{3}^{2}\right) \\
& +(i+j)\left(-3 x_{0}^{2}+x_{1}^{2}+x_{2}^{2}+x_{3}^{2}+2 x_{0}\right)+k\left(2 x_{0}-1\right), \\
h_{2}(x)= & \widetilde{\mathcal{Z}}_{2}(x)-\widetilde{\mathcal{Z}}_{1}(x)(i+j+1)+\widetilde{\mathcal{Z}}_{0}(x)(i+j+k) \\
= & \left(3 x_{0}^{2}-x_{1}^{2}-x_{2}^{2}-x_{3}^{2}\right)-2(i+j+1) x_{0}+i+j+k .
\end{aligned}
$$

Their restrictions to $\mathbb{S}^{3}$ are the quaternionic-valued spherical harmonics

$$
\begin{aligned}
& h_{1 \mid \mathbb{S}^{3}}(x)=\left(8 x_{0}^{3}-4 x_{0}^{2}-4 x_{0}+1\right)+(i+j)\left(-4 x_{0}^{2}+2 x_{0}+1\right)+k\left(2 x_{0}-1\right), \\
& h_{2 \mid \mathbb{S}^{3}}(x)=\left(4 x_{0}^{2}-1\right)-2(i+j+1) x_{0}+i+j+k .
\end{aligned}
$$

\section{An Almansi Decomposition for Slice-regular Functions}

Polynomials $P(x)=\sum_{k} x^{k} a_{k}$ with quaternionic coefficients do not belong to the kernel of the Cauchy-Riemann-Fueter operator. Instead they are examples of slice-regular functions. This function theory was introduced in 2006-2007 by Gentili and Struppa [6] for functions of one quaternionic variable. We refer to $[5,7-9]$ for precise definitions and for more properties and results about this class of functions. Here we recall only the basic facts that are necessary to state and prove the Almansi decomposition for slice-regular functions.

Slice function theory is based on the "slice" decomposition of $\mathbb{H}$. For each quaternion $J$ in the sphere of imaginary units

$$
\mathbb{S}_{\mathbb{H}}=\left\{J \in \mathbb{H} \mid J^{2}=-1\right\}=\left\{x_{1} i+x_{2} j+x_{3} k \in \mathbb{H} \mid x_{1}^{2}+x_{2}^{2}+x_{3}^{2}=1\right\},
$$

let $\mathbb{C}_{J}=\langle 1, J\rangle \simeq \mathbb{C}$ be the subalgebra generated by $J$. Then

$$
\mathbb{H}=\bigcup_{J \in \mathbb{S}_{\mathbb{H}}} \mathbb{C}_{J}, \quad \text { with } \mathbb{C}_{J} \cap \mathbb{C}_{K}=\mathbb{R} \quad \text { for every } J, K \in \mathbb{S}_{\mathbb{H}}, \quad J \neq \pm K .
$$

A differentiable function $f: \Omega \subseteq \mathbb{H} \rightarrow \mathbb{H}$ is called (left) slice-regular [6] on the open set $\Omega$ if, for each $J \in \mathbb{S}_{\mathbb{H}}$, the restriction $f_{\mid \Omega \cap \mathbb{C}_{J}}: \Omega \cap \mathbb{C}_{J} \rightarrow \mathbb{H}$ is holomorphic with respect to the complex structure defined by left multiplication by $J$. 


\subsection{Slice Functions}

Let $D \subseteq \mathbb{C}$, invariant with respect to conjugation. If a function $F: D \subseteq \mathbb{C} \rightarrow$ $\mathbb{H} \otimes \mathbb{C}$ satisfies $F(\bar{z})=\overline{F(z)}$ for every $z \in D$, then $F$ is called a stem function on $D$. Let $\Omega_{D}=\cup_{J \in \mathbb{S}_{\mathbb{H}}} \Phi_{J}(D) \subset \mathbb{H}$, where for any $J \in \mathbb{S}_{\mathbb{H}}$, the map $\Phi_{J}$ : $\mathbb{C} \rightarrow \mathbb{C}_{J}$ is the canonical isomorphism defined by $\Phi_{J}(a+i b):=a+J b$. Open domains in $\mathbb{H}$ of the form $\Omega=\Omega_{D}$ are called circular (or axially symmetric) domains.

The stem function $F=F_{1}+i F_{2}$ on $D$ induces the (left) slice function $f=\mathcal{I}(F): \Omega_{D} \rightarrow \mathbb{H}$ as follows: if $x=\alpha+J \beta=\Phi_{J}(z) \in \Omega_{D} \cap \mathbb{C}_{J}$, then

$$
f(x):=F_{1}(z)+J F_{2}(z) .
$$

Slice functions were introduced in [7]. They are exactly the quaternionic functions compatible with the slice structure of the skew-algebra $\mathbb{H}$, i.e., the ones corresponding to a commutative diagram for every $J \in \mathbb{S}_{\mathbb{H}}$

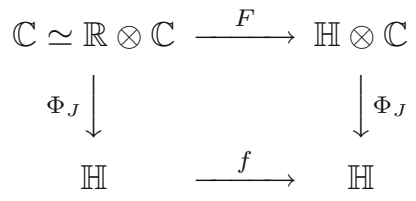

The slice function $f$ is (left) slice-regular if and only if $F$ is holomorphic. If the domain $D$ intersects the real axis, this definition of slice regularity is equivalent to the one proposed by Gentili and Struppa [6].

The function $f=\mathcal{I}(F)$ is called slice-preserving if $F_{1}$ and $F_{2}$ are realvalued. This is equivalent to the condition $f(\bar{x})=\overline{f(x)}$ for every $x \in \Omega_{D}$.

\subsection{Operations on Slice Functions}

Let $\Omega=\Omega_{D}$ be an open circular domain in $\mathbb{H}$. The slice product of two slice functions $f=\mathcal{I}(F), g=\mathcal{I}(G)$ on $\Omega$ is defined by means of the pointwise product of the stem functions $F$ and $G$

$$
f \cdot g=\mathcal{I}(F G) \text {. }
$$

If $f$ is slice-preserving, then $f \cdot g$ coincides with the pointwise product of $f$ and $g$. If $f, g$ are slice-regular on $\Omega$, then also their slice product $f \cdot g$ is slice-regular on $\Omega$. For example, the polynomial $P(x)$ given in Example 3 is the slice product $P(x)=(x-1) \cdot(x-i) \cdot(x-j)$.

The slice derivatives $\frac{\partial f}{\partial x}, \frac{\partial f}{\partial x^{c}}$ of a slice functions $f=\mathcal{I}(F)$ are defined by means of the complex Cauchy-Riemann operators

$$
\frac{\partial f}{\partial x}=\mathcal{I}\left(\frac{\partial F}{\partial z}\right), \quad \frac{\partial f}{\partial x^{c}}=\mathcal{I}\left(\frac{\partial F}{\partial \bar{z}}\right) .
$$

Note that $f$ is slice-regular on $\Omega$ if and only if $\frac{\partial f}{\partial x^{c}}=0$ and if $f$ is slice-regular on $\Omega$ then also $\frac{\partial f}{\partial x}$ is slice-regular on $\Omega$.

We recall other two useful concepts introduced in [7]. The function $f_{s}^{\circ}$ : $\Omega \rightarrow \mathbb{H}$, called spherical value of $f$, and the function $f_{s}^{\prime}: \Omega \backslash \mathbb{R} \rightarrow \mathbb{H}$, called spherical derivative of $f$, are defined as

$$
f_{s}^{\circ}(x):=\frac{1}{2}(f(x)+f(\bar{x})) \quad \text { and } \quad f_{s}^{\prime}(x):=\frac{1}{2} \operatorname{Im}(x)^{-1}(f(x)-f(\bar{x})),
$$


where $\operatorname{Im}(x)=\frac{1}{2}(x-\bar{x})=i x_{1}+j x_{2}+k x_{3}$. The functions $f_{s}^{\circ}$ and $f_{s}^{\prime}$ are slice functions, constant on 2-spheres $\mathbb{S}_{x}=\alpha+\mathbb{S}_{\mathbb{H}} \beta$ for any $x=\alpha+J \beta \in \Omega \backslash \mathbb{R}$, such that

$$
f(x)=f_{s}^{\circ}(x)+\operatorname{Im}(x) f_{s}^{\prime}(x),
$$

for every $x \in \Omega \backslash \mathbb{R}$. For any slice-regular function $f$ on $\Omega, f_{s}^{\prime}$ extends as the slice derivative $\frac{\partial f}{\partial x}$ on $\Omega \cap \mathbb{R}$. Note that for each $x \in \Omega \backslash \mathbb{R}, f_{s}^{\circ}(x)$ is the spherical mean of $f$ on $\mathbb{S}_{x}$, while $f_{s}^{\prime}(x)$ is the spherical mean of the function $\operatorname{Im}(x)^{-1} f(x)$ on $\mathbb{S}_{x}$.

\subsection{Almansi Type Decomposition}

We recall a result proved in [13, Corollary 3.6.2 and Theorem 3.6.3] about some formulas linking the spherical value and the spherical derivative of slice functions with the Cauchy-Riemann-Fueter operator.

Theorem 3. ([13]). Let $\Omega$ be an open circular domain in $\mathbb{H}$. Let $f: \Omega \rightarrow \mathbb{H}$ be a slice function of class $\mathcal{C}^{1}(\Omega)$. Then

1. $f$ is slice-regular if and only if $\bar{\partial}_{C R F} f=-f_{s}^{\prime}$.

2. If $f: \Omega \rightarrow \mathbb{H}$ is slice-regular, then it holds:

(a) The four real components of $f_{s}^{\prime}$ are harmonic on $\Omega$.

(b) $\Delta f_{s}^{\circ}=-2 \frac{\partial f_{s}^{\prime}}{\partial x_{0}}$.

(c) The following generalization of Fueter's Theorem holds:

$$
\bar{\partial}_{C R F} \Delta f=\Delta \bar{\partial}_{C R F} f=-\Delta f_{s}^{\prime}=0
$$

and therefore $f$ is biharmonic.

We are now able to generalize Theorem 2 to every slice-regular function.

Theorem 4. (Quaternionic Almansi Theorem). Let $f$ be slice-regular on a circular set $\Omega$. There exist two unique quaternionic-valued zonal harmonic functions $h_{1}, h_{2}$ with pole 1 , such that

$$
f(x)=h_{1}(x)-\bar{x} h_{2}(x) \quad \forall x \in \Omega .
$$

The functions $h_{1}$ and $h_{2}$ can be computed through differentiation:

$$
h_{1}=-\bar{\partial}_{C R F}(x f), \quad h_{2}=-\bar{\partial}_{C R F} f .
$$

Moreover, $f$ is slice-preserving if and only if $h_{1}$ and $h_{2}$ are real-valued.

Proof. If $f: \Omega \rightarrow \mathbb{H}$ is slice-regular, then it holds, from (3),

$$
f(x)=f_{s}^{\circ}(x)+\operatorname{Im}(x) f_{s}^{\prime}(x)=\left(f_{s}^{\circ}(x)+x_{0} f_{s}^{\prime}(x)\right)-\bar{x} f_{s}^{\prime}(x),
$$

with $h_{1}(x):=f_{s}^{\circ}(x)+x_{0} f_{s}^{\prime}(x)$ and $h_{2}(x):=f_{s}^{\prime}(x)$ slice functions on $\Omega$, axially symmetric with respect to the real axis. From point 2(a) of Theorem 3 the function $h_{2}$ is harmonic on $\Omega$. A direct computation gives

$$
\Delta\left(h_{1}\right)=\Delta\left(f_{s}^{\circ}\right)+2 \frac{\partial f_{s}^{\prime}}{\partial x_{0}}+x_{0} \Delta\left(f_{s}^{\prime}\right) .
$$


From point 2(b) of Theorem 3 it follows that also the function $h_{1}$ is harmonic on $\Omega$. It can also be observed, using the Leibniz-type formula for spherical derivative (see $[7, \S 5]$ ), that

$$
(x f)_{s}^{\prime}=(x \cdot f)_{s}^{\prime}=x_{s}^{\prime} f_{s}^{\circ}+x_{s}^{\circ} f_{s}^{\prime}=f_{s}^{\circ}+x_{0} f_{s}^{\prime}=h_{1} .
$$

Since also the slice product $x \cdot f$ is slice-regular, this gives again the harmonicity of $h_{1}$. In view of point 1 of Theorem $3, h_{1}=(x f)_{s}^{\prime}=-\bar{\partial}_{C R F}(x f)$ and $h_{2}=f_{s}^{\prime}=-\bar{\partial}_{C R F} f$. Since $f$ is slice-preserving if and only if $f_{s}^{\circ}$ and $f_{s}^{\prime}$ are real-valued, and since $h_{1}(x)=f_{s}^{\circ}(x)+x_{0} f_{s}^{\prime}(x), h_{2}(x)=f_{s}^{\prime}(x)$, this condition is equivalent to having $h_{1}$ and $h_{2}$ real-valued.

It remains to prove that $h_{1}$ and $h_{2}$ are uniquely determined by $f$. Assume that $f(x)=h_{1}(x)-\bar{x} h_{2}(x)$ with $h_{1}, h_{2}$ axially symmetric with respect to the real axis. A direct computation gives $f_{s}^{\prime}(x)=h_{2}(x)$ for every $x \in \Omega \backslash \mathbb{R}$ and then $h_{1}(x)=f(x)+\bar{x} f_{s}^{\prime}(x)$.

We now prove a converse of the preceding theorem.

Theorem 5. Let $h_{1}$ and $h_{2}$ be $\mathbb{H}$-valued axially symmetric functions on $\Omega$, of class $\mathcal{C}^{1}$. Then $g:=h_{1}-\bar{x} h_{2}$ is a slice function on $\Omega$. The function $g$ is slice-regular if and only if $h_{1}$ and $h_{2}$ satisfy the equations

$$
\left\{\begin{array}{l}
\partial_{\alpha} h_{1}-\alpha \partial_{\alpha} h_{2}-\beta \partial_{\beta} h_{2}=2 h_{2} \\
\partial_{\beta} h_{1}-\alpha \partial_{\beta} h_{2}+\beta \partial_{\alpha} h_{2}=0
\end{array}\right.
$$

where $\alpha=x_{0}, \beta=|\operatorname{Im}(x)|$. In this case, $h_{1}$ and $h_{2}$ are zonal harmonic.

Proof. Let $\Omega=\Omega_{D}$. In view of the symmetry of $h_{1}$ and $h_{2}$, given $z=x_{0}+i \beta \in$ $D$ and $x=x_{0}+J \beta \in \Omega$, the function

$$
G(z)=G_{1}(z)+i G_{2}(z)
$$

with $G_{1}(z):=h_{1}(x)-x_{0} h_{2}(x)$ and $G_{2}(z)=|\operatorname{Im}(x)| h_{2}(x)$, is a $\mathcal{C}^{1}$ stem function on $D$ that induces the slice function $g$. Equation (4) for $h_{1}, h_{2}$ are equivalent to the Cauchy-Riemann equations for $G$. The last statement follows from the uniqueness of the harmonic decomposition given in Theorem 4 .

Example 4. Consider again the polynomial $P(x)=x^{2}$ of Example 2. According to Theorem 4, we can obtain the harmonic functions $h_{1}, h_{2}$ applying the Cauchy-Riemann-Fueter operator. Since

$\bar{\partial}_{C R F} P=\bar{\partial}_{C R F}\left(x^{2}\right)=-2 x_{0}, \quad \bar{\partial}_{C R F}(x P)=\bar{\partial}_{C R F}\left(x^{3}\right)=-3 x_{0}^{2}+x_{1}^{2}+x_{2}^{2}+x_{3}^{2}$, then $h_{1}(x)=3 x_{0}^{2}-x_{1}^{2}-x_{2}^{2}-x_{3}^{2}$ and $h_{2}(x)=2 x_{0}$.

Example 5. Let $f(x)=\log (x)$ be the slice-preserving slice-regular function on $\Omega=\mathbb{H} \backslash\{x \in \mathbb{R} \mid x \leq 0\}$ induced by the (principal) complex logarithm

$$
f(x)=\log (x)= \begin{cases}\log |x|+\frac{\operatorname{Im}(x)}{|\operatorname{Im}(x)|} \arccos \left(\frac{\operatorname{Re}(x)}{|x|}\right) & \text { if } x \notin \mathbb{R}, \\ \log |x| \quad \text { if } x \in \mathbb{R}, & \end{cases}
$$


with $\arccos \left(\frac{\operatorname{Re}(x)}{|x|}\right) \in[0, \pi]$. Then $f(x)=h_{1}(x)-\bar{x} h_{2}(x)$ on $\Omega$, with real harmonic components

$$
\left\{\begin{array}{l}
h_{1}(x)=\frac{1}{2} \log \left(x_{0}^{2}+x_{1}^{2}+x_{2}^{2}+x_{3}^{2}\right)+\frac{x_{0}}{\sqrt{x_{1}^{2}+x_{2}^{2}+x_{3}^{2}}} \arccos \left(\frac{x_{0}}{\sqrt{x_{0}^{2}+x_{1}^{2}+x_{2}^{2}+x_{3}^{2}}}\right), \\
h_{2}(x)=\frac{1}{\sqrt{x_{1}^{2}+x_{2}^{2}+x_{3}^{2}}} \arccos \left(\frac{x_{0}}{\sqrt{x_{0}^{2}+x_{1}^{2}+x_{2}^{2}+x_{3}^{2}}}\right) .
\end{array}\right.
$$

Observe that $h_{1}$ extends continuously to the real points $x_{0} \in\{x \in \mathbb{R} \mid x>0\}$ as $\log \left(x_{0}\right)+1$, while $h_{2}$ extends to the same set as $1 / x_{0}$.

\section{Mean Value Property and Poisson Formula}

As an application of the quaternionic Almansi Theorem, we give a mean value property for slice-regular functions. Let $\sigma$ denote the normalized rotationinvariant surface-area measure on the unit sphere $\mathbb{S}^{3}$ of $\mathbb{H} \simeq \mathbb{R}^{4}$, such that $\sigma\left(\mathbb{S}^{3}\right)=1$.

Proposition 2. (Mean value formula). Let $f$ be slice-regular on a circular set $\Omega$. Assume that the open ball $B(a, r)$ with centre $a \in \Omega$ and radius $r$ has closure $\overline{B(a, r)}$ contained in $\Omega$. Then

$$
f(a)=\int_{\mathbb{S}^{3}} f(a+r \zeta) d \sigma(\zeta)+r \int_{\mathbb{S}^{3}} \bar{\zeta} f_{s}^{\prime}(a+r \zeta) d \sigma(\zeta) .
$$

Proof. Let $f=h_{1}-\bar{x} h_{2}$ be the Almansi decomposition of $f$. From the mean value formula applied to the harmonic real components of $h_{1}$ and $h_{2}$

$$
h_{1}(a)=\int_{\mathbb{S}^{3}} h_{1}(a+r \zeta) d \sigma(\zeta), \quad h_{2}(a)=\int_{\mathbb{S}^{3}} h_{2}(a+r \zeta) d \sigma(\zeta) .
$$

Therefore

$$
\begin{aligned}
f(a) & =h_{1}(a)-\bar{a} h_{2}(a)=\int_{\mathbb{S}^{3}}\left(h_{1}(a+r \zeta)-\bar{a} h_{2}(a+r \zeta)\right) d \sigma(\zeta) \\
& =\int_{\mathbb{S}^{3}}\left(h_{1}(a+r \zeta)-\overline{(a+r \zeta)} h_{2}(a+r \zeta)+r \bar{\zeta} h_{2}(a+r \zeta)\right) d \sigma(\zeta) \\
& =\int_{\mathbb{S}^{3}}\left(f(a+r \zeta)+r \bar{\zeta} f_{s}^{\prime}(a+r \zeta)\right) d \sigma(\zeta),
\end{aligned}
$$

and the mean value formula is proved.

A slice-regular function $f$, being biharmonic, satisfies also a mean value formula of the form

$$
f(a)=\int_{\mathbb{S}^{3}} f(a+r \zeta) d \sigma(\zeta)-\frac{r^{2}}{8} \Delta f(a)
$$

(see, e.g., [11, Chap. 7]). Comparing this formula with the one obtained before, we get the following.

Corollary 1. Let $f$ be slice-regular on a circular set $\Omega$. Assume that the open ball $B(a, r)$ with centre $a \in \Omega$ and radius $r$ has closure contained in $\Omega$. Then

$$
\Delta f(a)=-\frac{8}{r} \int_{\mathbb{S}^{3}} \bar{\zeta} f_{s}^{\prime}(a+r \zeta) d \sigma(\zeta) .
$$


Let $P(x, \zeta)=\left(1-|x|^{2}\right) /|x-\zeta|^{4}$ be the Poisson kernel of the unit ball $\mathbb{B}$ in $\mathbb{R}^{4}$.

Proposition 3. (Poisson formula). Let $f$ be slice-regular on a circular set $\Omega$. Let the open ball $B(a, r)$ have closure contained in $\Omega$. Then, for every $x \in \mathbb{B}$, it holds

$f(a+r x)=\int_{\mathbb{S}^{3}} f(a+r \zeta) P(x, \zeta) d \sigma(\zeta)+r \int_{\mathbb{S}^{3}}(\bar{\zeta}-\bar{x}) f_{s}^{\prime}(a+r \zeta) P(x, \zeta) d \sigma(\zeta)$.

Proof. We can proceed as in the proof of Proposition 2, using the Poisson formula for the quaternionic-valued harmonic components $h_{1}$ and $h_{2}$ of $f$.

Observe that the Poisson kernel itself is related to the spherical derivative of a slice-regular function, the quaternionic Koebe function $f(x)=$ $(1-x)^{-2} x$ (see [13, Cor. 3.6.7]).

When the centre $a$ is a real point, the ball $B(a, r)$ is a circular set. In this case we can obtain two formulas in which the spherical derivative of $f$ does not appear.

Corollary 2. (Axially symmetric mean value and Poisson formulas). Let $f$ be slice-regular on a circular set $\Omega$. Assume that the open ball $B(a, r)$ with real centre $a \in \mathbb{R}$ and radius $r$ has closure contained in $\Omega$. Then it holds

$$
f(a)=\int_{\mathbb{S}^{3}}\left(1+r(\zeta+\bar{\zeta})(\zeta-\bar{\zeta})^{-1}\right) f(a+r \zeta) d \sigma(\zeta)
$$

$$
\begin{aligned}
f(a+r x)= & \int_{\mathbb{S}^{3}}\left(1+r((\bar{\zeta}-\bar{x}) P(x, \zeta)+(\zeta-\bar{x}) P(x, \bar{\zeta}))(\zeta-\bar{\zeta})^{-1}\right) \\
& f(a+r \zeta) d \sigma(\zeta),
\end{aligned}
$$

for every $x \in \mathbb{B}$.

Proof. We transform the second integral of the mean value formula of Proposition 2. Since $a$ is real, it holds

$$
\bar{\zeta} f_{s}^{\prime}(a+r \zeta)=\bar{\zeta}(\zeta-\bar{\zeta})^{-1}(f(a+r \zeta)-f(a+r \bar{\zeta}))
$$

By symmetry w.r.t. conjugation, we get

$$
\begin{aligned}
\int_{\mathbb{S}^{3}} \bar{\zeta} f_{s}^{\prime}(a+r \zeta) d \sigma(\zeta)= & \int_{\mathbb{S}^{3}} \bar{\zeta}(\zeta-\bar{\zeta})^{-1} f(a+r \zeta) d \sigma(\zeta) \\
& +\int_{\mathbb{S}^{3}} \zeta(\zeta-\bar{\zeta})^{-1} f(a+r \zeta) d \sigma(\zeta) \\
= & \int_{\mathbb{S}^{3}}(\zeta+\bar{\zeta})(\zeta-\bar{\zeta})^{-1} f(a+r \zeta) d \sigma(\zeta)
\end{aligned}
$$

This proves point 1 . For the second point, we transform as above the second integral of the formula of Proposition 3

$$
\int_{\mathbb{S}^{3}}(\bar{\zeta}-\bar{x}) f_{s}^{\prime}(a+r \zeta) P(x, \zeta) d \sigma(\zeta)
$$




$$
\begin{aligned}
= & \int_{\mathbb{S}^{3}}(\bar{\zeta}-\bar{x})(\zeta-\bar{\zeta})^{-1} P(x, \zeta) f(a+r \zeta) d \sigma(\zeta) \\
& +\int_{\mathbb{S}^{3}}(\zeta-\bar{x})(\zeta-\bar{\zeta})^{-1} P(x, \bar{\zeta}) f(a+r \zeta) d \sigma(\zeta) .
\end{aligned}
$$

Open Access. This article is licensed under a Creative Commons Attribution 4.0 International License, which permits use, sharing, adaptation, distribution and reproduction in any medium or format, as long as you give appropriate credit to the original author(s) and the source, provide a link to the Creative Commons licence, and indicate if changes were made. The images or other third party material in this article are included in the article's Creative Commons licence, unless indicated otherwise in a credit line to the material. If material is not included in the article's Creative Commons licence and your intended use is not permitted by statutory regulation or exceeds the permitted use, you will need to obtain permission directly from the copyright holder. To view a copy of this licence, visit http:// creativecommons.org/licenses/by/4.0/.

Publisher's Note Springer Nature remains neutral with regard to jurisdictional claims in published maps and institutional affiliations.

Funding Open access funding provided by Universitá degli Studi di Trento within the CRUI-CARE Agreement.

\section{References}

[1] Almansi, E.: Sull'integrazione dell'equazione differenziale $\Delta^{2 n}=0$. Annali di Mat. (3) 2, 1-51 (1899)

[2] Axler, S., Bourdon, P., Ramey, W.: Harmonic function theory, volume 137 of graduate texts in mathematics, 2nd edn. Springer-Verlag, New York (2001)

[3] Colombo, F., Sabadini, I., Struppa, D.C.: Slice monogenic functions. Israel J. Math. 171, 385-403 (2009)

[4] Faustino, N., Ren, G.: (Discrete) Almansi type decompositions: an umbral calculus framework based on osp(1|2) symmetries. Math. Methods Appl. Sci. 34(16), 1961-1979 (2011)

[5] Gentili, G., Stoppato, C., Struppa, D.C.: Regular functions of a quaternionic variable. Springer monographs in mathematics. Springer, Berlin (2013)

[6] Gentili, G., Struppa, D.C.: A new theory of regular functions of a quaternionic variable. Adv. Math. 216(1), 279-301 (2007)

[7] Ghiloni, R., Perotti, A.: Slice regular functions on real alternative algebras. Adv. Math. 226(2), 1662-1691 (2011)

[8] Ghiloni, R., Perotti, A., Stoppato, C.: The algebra of slice functions. Trans. Amer. Math. Soc. 369(7), 4725-4762 (2017)

[9] Ghiloni, R., Perotti, A., Stoppato, C.: Division algebras of slice functions. Proc. R. Soc. Edinburgh 150(4), 2055-2082 (2020). https://doi.org/10.1017/ prm.2019.13 
[10] Malonek, H.R., Ren, G.: Almansi-type theorems in Clifford analysis. Math. Methods Appl. Sci. 25(16-18), 1541-1552 (2002)

[11] Mitrea, D.: Distributions, partial differential equations, and harmonic analysis, 2nd edn. Universitext Springer, Cham (2018)

[12] Perotti, A.: A four dimensional Bernstein theorem. (2019) arXiv:1903.03068, submitted

[13] Perotti, A.: Slice regularity and harmonicity on Clifford algebras. In: Topics in Clifford Analysis - Special Volume in Honor of Wolfgang Sprößig. Trends Math. Springer, Basel (2019) https://doi.org/10.1007/978-3-030-23854-4

[14] Perotti, A.: Almansi-type theorems for slice-regular functions on Clifford algebras. Complex Variables and Elliptic Equations, (2020) https://doi.org/10. 1080/17476933.2020.1755967

[15] Ren, G.: Almansi decomposition for Dunkl operators. Sci. China Ser. A 48(Suppl), 333-342 (2005)

\author{
Alessandro Perotti \\ Department of Mathematics \\ University of Trento \\ Via Sommarive 14 \\ Trento \\ Italy \\ e-mail: alessandro.perotti@unitn.it
}

Received: January 26, 2020.

Accepted: July 11, 2020. 